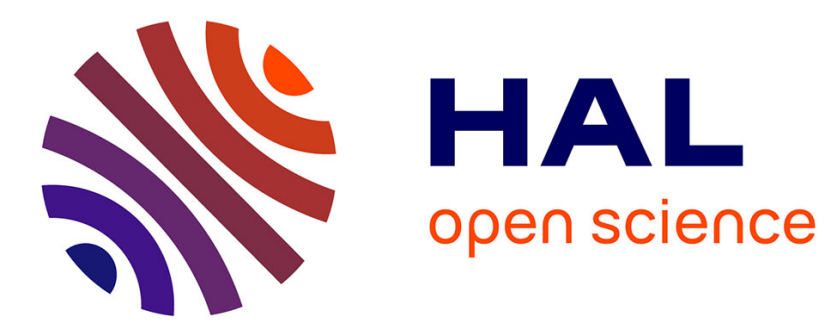

\title{
Myocardite aiguë dans les suites d'une infection à Salmonella enteritidis
}

J Brice, S Baumard, F Loeb, L Brasme, R Jaussaud, Y N'Guyen, Y N'guyen

\section{To cite this version:}

J Brice, S Baumard, F Loeb, L Brasme, R Jaussaud, et al.. Myocardite aiguë dans les suites d'une infection à Salmonella enteritidis. Médecine et Maladies Infectieuses, 2013, 43, pp.248 - 250. 10.1016/j.medmal.2013.05.002 . hal-03267223

\section{HAL Id: hal-03267223 https://hal.science/hal-03267223}

Submitted on 28 Jun 2021

HAL is a multi-disciplinary open access archive for the deposit and dissemination of scientific research documents, whether they are published or not. The documents may come from teaching and research institutions in France or abroad, or from public or private research centers.
L'archive ouverte pluridisciplinaire $\mathbf{H A L}$, est destinée au dépôt et à la diffusion de documents scientifiques de niveau recherche, publiés ou non, émanant des établissements d'enseignement et de recherche français ou étrangers, des laboratoires publics ou privés. 
Myocardite aiguë dans les suites d'une infection à Salmonella

Enteritidis.

Salmonella Enteritidis infection complicated by acute myocarditis.

(1259 mots)

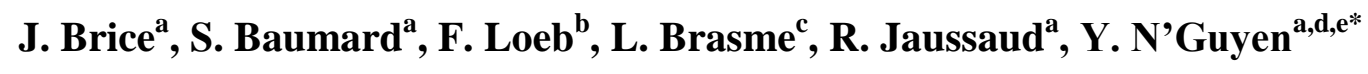

${ }^{\text {a }}$ Service de Médecine Interne, Maladies Infectieuses et Immunologie clinique, Hôpital Robert Debré CHU Reims, Avenue général Koenig 51100 Reims

${ }^{\mathrm{b}}$ Service de Cardiologie, Hôpital Robert Debré CHU Reims, Avenue général Koenig 51100 Reims

${ }^{\mathrm{c}}$ Laboratoire de Bactériologie, Hôpital Robert Debré CHU Reims, Avenue général Koenig 51100 Reims

${ }^{\mathrm{d}}$ Laboratoire de Virologie médicale et moléculaire, Hôpital Robert Debré CHU Reims, Avenue général Koenig 51100 Reims

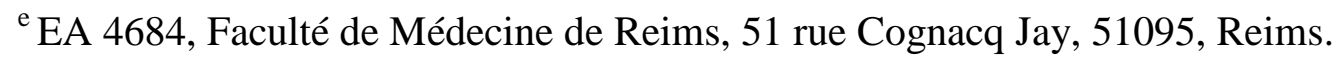

* auteur correspondant. Service de Médecine Interne, Maladies Infectieuses et Immunologie clinique, Hôpital Robert Debré CHU Reims, Avenue général Koenig 51100 Reims. Tél : 0326-78-94-22. Fax 03-26-78-40-90. mail : yohan.nguyen@wanadoo.fr 


\section{Résumé: Aucun}

Mots clés : myocardite, Salmonella Enteritidis

\section{Abstract : None}

Keywords : myocarditis, Salmonella Enteritidis 


\section{Introduction :}

Les salmonelloses mineures restent une cause très fréquente de diarrhées infectieuses dans les pays développés [1] et elles ne sont classiquement pourvoyeuses de bactériémies que chez l'immunodéprimé et notamment le patient séropositif pour le virus de l'immunodéficience humaine (VIH) [2]. Contrairement à Salmonella Typhi et Paratyphi, leur implication dans la survenue de myocardites létales ou non est beaucoup moins documentée [3-8].

Nous rapportons un cas de myocardite aiguë d'évolution favorable dans les suites immédiates d'une diarrhée infectieuse à Salmonella Enteritidis survenue en France chez un patient de 16 ans sans déficit immunitaire.

\section{Présentation du cas :}

Un homme de 16 ans, sans aucun antécédent, est hospitalisé pour des diarrhées non glairo-sanglantes évoluant depuis 36 heures associées à de la fièvre et des frissons. L'interrogatoire ne retrouvait pas de voyage à l'étranger dans les six derniers mois, pas de cas similaires dans l'entourage ni de prise médicamenteuse ou d'antibiothérapie préalable. Aucune symptomatologie digestive n'avait été rapportée durant les neuf mois précédents, on retrouvait juste la notion de consommation de poulet dans un restaurant chinois avec sa famille la veille du début des symptômes.

À l'admission (J0), le patient était en sepsis (température $40.3^{\circ} \mathrm{C}$, pouls $105 / \mathrm{min}$, tension artérielle 90/50 $\mathrm{mm} \mathrm{Hg}$, leucocytes 17100/mm3 dont 15500 polynucléaires neutrophiles). L'examen clinique initial ne retrouvait qu'une sensibilité diffuse de l'abdomen 
sans défense. Le bilan biologique initial était sans particularité hormis une protéine C-réactive à $157 \mathrm{mg} / \mathrm{L}(\mathrm{N}<10 \mathrm{mg} / \mathrm{L})$ et une procalcitonine à $0,95 \mu \mathrm{g} / \mathrm{L}(\mathrm{N}<0,50 \mu \mathrm{g} / \mathrm{L})$.

Une antibiothérapie probabiliste par ceftriaxone a été débutée après réalisation d'une coproculture. Celle-ci retrouvait l'absence de leucocytes mais de rares colonies de Salmonella Enteritidis sensibles à l'amoxicilline. L'évolution clinique a été rapidement favorable avec apyrexie dès le second jour d'antibiothérapie, prévue pour une durée totale de 5 jours (figure 1A). Les hémocultures sont demeurées stériles.

Aux troisième et quatrième jours, le patient a présenté des épisodes de douleur thoracique rétro-sternale constrictive augmentée à l'inspiration profonde spontanément régressive sous 30 minutes. Ces douleurs se sont associées à l'apparition d'un bloc de branche droit incomplet sur l'électrocardiogramme et à une élévation des troponines cardiaques supérieure à $1 \mu \mathrm{g} / \mathrm{L}$ (figure $1 \mathrm{~A})$.

Le patient a été transféré pendant 24 heures en unité de soins intensifs de cardiologie où la surveillance scopée n'a isolé aucun trouble du rythme. L’échographie cardiaque y était normale avec un péricarde sec, des cavités non dilatées, une fraction d'éjection du ventricule gauche (FEVG) estimée à 68\% (Simpson) sans trouble de la cinétique segmentaire. Une coronarographie n'a pas été réalisée compte tenu du jeune âge du patient, de l'absence de facteurs de risque cardiovasculaire personnels ou familiaux et de l'absence de troubles de repolarisation significatifs dans des dérivations compatibles avec un territoire vasculaire. Devant ce tableau, le diagnostic de myocardite aiguë a été retenu même si aucune biopsie endomyocardique n'a été réalisée. L'indication d'un traitement par béta-bloquants ou par inhibiteurs de l'enzyme de conversion n'a pas été retenue compte-tenu de l'absence de trouble du rythme ventriculaire et de dysfonction ventriculaire gauche respectivement.

L'évolution a été favorable spontanément, après une hospitalisation de 8 jours, jusqu'à négativation des troponines cardiaques. Aucune autre étiologie pourvoyeuse de myocardite, 
auto-immune ou infectieuse (Parvovirus B19, Coxsackie B, Human Herpes Virus 6, Cytomegalovirus, Epstein Barr Virus et Toxoplasma gondii), n'a été retrouvée et Salmonella Enteritidis a été considérée comme l'agent responsable de cette diarrhée infectieuse et de cette myocardite. La recherche d'une immunodépression associée a été infructueuse : sérologie VIH négative, absence de cytopénie et de corps de Jolly sur le frottis sanguin, phénotypage des lymphocytes circulants normal et dosage pondéral des immunoglobulines normal à l'exception d'une valeur normale basse d'Immunoglobulines $\mathrm{A}(\operatorname{Ig} \mathrm{A})$ totales à $1,10 \mathrm{~g} / \mathrm{L}$ à deux reprises $(\mathrm{N}<1,30 \mathrm{~g} / \mathrm{L}$ dans notre laboratoire, $0,80 \mathrm{~g} / \mathrm{L}$ pour d'autres).

Un mois après l'épisode, le patient était apyrétique, en bon état général et avait repris sa scolarité. La coproculture de contrôle était négative. Son ECG était inchangé. L’imagerie par résonance magnétique (IRM) cardiaque retrouvait une FEVG normale et une prise de contraste inféro-basale nodulaire intramyocardique évocatrice d'une séquelle de myocardite (figure 1B). Une épreuve d'effort a été réalisée de principe devant une dyspnée survenant exclusivement durant les épreuves de natation à son lycée et elle s'est avérée négative.

\section{Discussion:}

Nous rapportons un épisode de myocardite aiguë d'évolution favorable chez un jeune homme de 16 ans dans les suites immédiates d'une diarrhée infectieuse à Salmonella Enteritidis. La biopsie endomyocardique qui aurait pu, avec certitude, confirmer le diagnostic de myocardite et la présence éventuelle de Salmonella spp [4] n'a pas été réalisée ici, en l'absence de défaillance cardiaque et ce, conformément aux recommandations en vigueur [9]. Nous retenons toutefois Salmonella Enteritidis comme l'agent vraisemblable de cette myocardite compte tenu de la survenue de cette myocardite au décours immédiat d'un épisode 
de diarrhée infectieuse documentée bactériologiquement, de l'absence d'autre étiologie plausible aux vues du contexte et de l'imputabilité bibliographique [3-8].

La coproculture pourrait-être interprétée isolément du fait de l'absence de leucocytes comme un portage chronique de Salmonella Enteritidis sans rapport avec une myocardite aiguë idiopathique. Toutefois, l'absence de syndrome gastro-entéritique dans les 9 mois précédents, l'amélioration clinico-biologique du tableau initial de diarrhée infectieuse avec sepsis sous antibiothérapie (figure 1A) et le caractère invasif de Salmonella Enteritidis parmi les salmonelles mineures plaident contre un simple portage chronique [2]. L'absence de leucocytes peut être liée au fait que la coproculture a été réalisée très précocement après le début des symptômes et cet élément n'est d'ailleurs pas rapporté dans les autres cas publiés d'évolution favorable, repris dans la revue de la littérature de Papamichalis et collègues [4]. Parmi ces derniers, où une biopsie endomyocardique n'était pas réalisée, il n'y avait jamais de documentation bactériologique de certitude, à l'inverse de toutes les observations létales de la littérature [4].

Nous avons dans un premier temps recherché sans succès un déficit immunitaire chez ce jeune patient, devant le caractère inhabituel de cette complication alors que Salmonella Enteritidis et les salmonelloses mineures sont des pathogènes très fréquents [1]. Le dosage dans les limites inférieures de la normale des IgA à deux reprises chez notre patient ne rentre pas dans le cadre d'un authentique déficit en $\operatorname{IgA}$, du fait de l'absence de symptômes cliniques compatibles. On peut toutefois se poser la question du rôle de cette valeur limite inférieure d'IgA dans la génèse de cette myocardite après infection digestive à Salmonella Enteritidis, car un modèle murin semble suggérer le rôle d'un déficit en IgA dans la survenue d'infections plus sévères à Salmonella Typhimurium [10]. En l'absence d'immunodépression, nous nous sommes ensuite interrogés sur l'absence de cas groupés dans la famille ayant consommé un repas dans le même restaurant. L'explication retenue après nouvel interrogatoire du patient et 
de son entourage était la présence d'un vraisemblable effet «inoculum», puisque notre patient a été le seul à avoir copieusement mangé du poulet ce soir là.

À ce jour, aucun facteur de virulence pouvant expliquer la physiopathologie de ces myocardites de l'immunocompétent n'a été mis en évidence parmi les souches de Salmonella Enteritidis. D'authentiques infections myocardiques invasives ont été décrites après bactériémie chez des sujets immunodéprimés ou fragiles [5,8] mais elles ne permettent pas de préjuger du mécanisme chez l'immunocompétent. Dans notre observation, on peut noter que les douleurs thoraciques qui ont permis de porter le diagnostic de myocardite sont survenues après plusieurs jours d'antibiothérapie, ce qui va contre un mécanisme invasif et plaide plus en faveur d'un mécanisme post-infectieux ou en faveur d'un relargage d'un composé bactérien à propriété cardiotoxique.

Conclusions : Cette observation de myocardite d'évolution favorable vraisemblablement due à Salmonella Enteritidis chez un sujet immunocompétent et l'existence de plusieurs observations analogues publiées soulève la question du mécanisme par lequel les Salmonelles mineures peuvent rarement générer une myocardite chez l'immunocompétent. D’autres études sont nécessaires pour mieux préciser ce mécanisme et définir la proportion des salmonelloses mineures parmi les étiologies de myocardites aiguës de l'immunocompétent, car la recherche de salmonellose mineure ne fait pas partie du bilan couramment réalisé en France devant une myocardite aiguë.

Remerciements : Dr Simon Le Hello, CNR Salmonella institut Pasteur.

Conflit d'intérêts : Aucun. 


\section{Références :}

1. Sánchez-Vargas FM, Abu-El-Haija MA, Gómez-Duarte OG. Salmonella infections: an update on epidemiology, management, and prevention. Travel Med Infect Dis. 2011;9:263-77.

2. Dhanoa A, Fatt QK. Non-typhoidal Salmonella bacteraemia: epidemiology, clinical characteristics and its' association with severe immunosuppression. Ann Clin Microbiol Antimicrob. 2009;8:15.

3. Childs L, Gupta S. Salmonella Enteritidis induced myocarditis in a 16-year-old girl. BMJ Case Rep. 2012; DOI:10.1136/bcr-2012-007628

4. Papamichalis P, Argyraki K, Papamichalis M, Loukopoulos A, Dalekos GN, Rigopoulou EI. Salmonella Enteritidis Infection Complicated by Acute Myocarditis : A Case Report et Review of literature. Cardiol Res Pract. 2011;DOI: $10.4061 / 2011 / 574230$.

5. Hibbert B, Costiniuk C, Hibbert R, Joseph P, Alanazi H, Simard T et al. Cardiovascular complications of Salmonella Enteritidis infection. Can J Cardiol. $2010 ; 26: 323-5$

6. Oziol E, Bonal J, Chauveau E, Talard P, Carli P, Chagnon A. Acute myocarditis in non-typhoid Salmonella infection. Arch Mal Cœur vaiss. 1995;88:99-101

7. Huppertz HI et Sandhage K. Salmonella Enteriditis in reactive carditis. Lancet. $1993 ; 342: 1488-9$

8. Martinez-Martinez L, Mesa E, Rodriguez JE, Sanchez MP, Ugarte J, Algora Weber A et al. Bacteremia associated with mycotic aneurysm of the transversal 
aortic arch and myocarditis caused by Salmonella Enteritidis. Enferm Infecc Microbiol Clin. 1989;7:97-9

9. Cooper L, Baughman KL, Feldman AM, Frustaci A, Jessup M, Kuhl U et al. The role of endomyocardial biopsy in the management of cardiovascular disease. Eur Heart J. 2007;28:3076-93

10. Wijburg OL, Uren TK, Simpfendorfer K, Johansen FE, Brandtzaeg P, Strugnell RA. Innate secretory antibodies protect against natural Salmonella Typhimurium infection. J Exp Med. 2006;203:21-26. 


\section{Légende des Figures :}

Figure 1A: Courbe de température et évolution des troponines Ic ainsi que de la protéine CRéactive durant l'hospitalisation.

Flèches noires: douleurs thoraciques.

Figure1A: Temperature curve and Troponine Ic and $\mathrm{C}$ reactive protein during hospitalisation. Black arrows: Chest pain.

Figure 1B: Imagerie par résonance magnétique cardiaque à 1 mois (séquence $\mathrm{T} 1$ avec injection de gadolinium).

Flèches blanches: discret hypersignal inférobasal (réhaussement tardif), considéré comme séquellaire de cet épisode de myocardite.

Figure1B : Cardiac magnetic resonance imaging one month later (T1 weighted sequence with gadolinium injection)

White arrows : Inferobasal hypersignal (late enhancement on the delayed acquisition), interpreted as sequelae of acute myocarditis. 